\title{
Competition between gelation and crystallisation of a peculiar multicomponent liquid system based on ammonium salts $\dagger$
}

\author{
Iti Kapoor, ${ }^{\ddagger a}$ Eva-Maria Schön, ${ }^{\ddagger a}$ Jürgen Bachl, ${ }^{a}$ Dennis Kühbeck, ${ }^{a}$ Carlos Cativiela, ${ }^{b}$ Subhadeep Saha, ${ }^{c}$ \\ Rahul Banerjee, ${ }^{c}$ Stefano Roelens, ${ }^{d}$ José Juan Marrero-Tellado*e and David Díaz Díaz*a,b
}

\author{
${ }_{5}$ Received (in $\left.X X X, X X X\right)$ Xth $X X X X X X X X X 20 X X$, Accepted Xth XXXXXXXXX 20XX \\ DOI: 10.1039/b000000x
}

\begin{abstract}
An exemplar competition between gelation and crystallisation phenomena was examined with an unusual synergistic multicomponent (organo)gelator solution (MGS), which consists of a well-defined methanolic solution of $(1 R, 2 R)$-1,2-diaminocyclohexane L-tartrate containing 2.4 equiv of concentrated hydrochloric

10 acid. The optimal composition of the MGS was determined through meticulous solubility, gelation and structural studies, which support a transient gelation mechanism based on the kinetic self-assembly of the tartrate salt driven by hydrogen-bonding interactions, involving ammonium nitrogen donors and hydroxyl oxygen acceptors, and electrostatic interactions. The hydrochloric acid is involved in the solubilisation of the salt through an ionic dissociation-exchange process, which ends up with the formation-precipitation of

$15(1 R, 2 R)$-1,2-diaminocyclohexane dihydrochloride. As a consequence, an irreversible destruction of the gel takes place, which indicates the metastable nature of this phase that cannot be accessed from the thermodynamically equilibrated state. Gelation of a variety of oxygenated and nitrogenated solvents with moderate polarity occurred efficiently using extremely low MGS concentrations at low temperatures, and the gel phase was confirmed by dynamic rheological measurements. Several features make the described

${ }_{20}$ MGS unique: (1) it is a multicomponent solution where each component and its stoichiometry plays a key role in the reproducible formation and stabilization of the gels; (2) it is formed by simple, small, and commercially available chiral building blocks (dissolved in a well-defined solvent system), which are easily amenable for further modifications; (3) the gelation phenomenon takes place efficiently at low temperature upon warming up the isotropic solution, conversely to the typical gel preparation protocol;

25 (4) the formed organogels are not thermoreversible despite the non-covalent interactions that characterize the 3D-network.
\end{abstract}

\section{Introduction}

In a fascinating journey from serendipity to rational design, materials made by means of gelation of solvents (organogels or 30 hydrogels in the case of organic solvents or water respectively) have received increasing attention over the last two decades, ${ }^{1}$ because of their unique supramolecular architectures and

\footnotetext{
anstitut für Organische Chemie, Universität Regensburg, Universitätsstr. 31, 93040 Regensburg, Germany. E-mail: David.Diaz@chemie.uni-regensburg.de; Fax: + 49941 9434121; Tel.: + 499419434373

${ }^{b}$ Departamento de Química Orgánica, ISQCH, Universidad de Zaragoza-CSIC, Pedro Cerbuna 12, 50009 Zaragoza, Spain

c Physical/Materials Chemistry Division, National Chemical Laboratory, Dr. Homi Bhabha Road, Pune-411008, India

${ }^{d}$ Istituto di Metodologie Chimiche (IMC), Consiglio Nazionale delle Ricerche (CNR), Dipartimento di Chimica, Università di Firenze, Via della Lastruccia 13, I-50019 Sesto Fiorentino, Firenze, Italy

e Instituto Universitario de Bio-Orgánica "Antonio González", Universidad de La Laguna, Astrofísico Francisco Sánchez 2, 38206 La Laguna, Tenerife, Spain

† Iti Kapoor and Eva-Maria Schön contributed equally to this work.

$\dagger$ Electronic Supplementary Information (ESI) available: Additional experimental details, figures and tables. See DOI: 10.1039/b000000x/
}

potential applications ${ }^{2}$ as functional soft materials in the fabrication of sensors, ${ }^{3}$ liquid crystallines, ${ }^{4}$ electrophoretic and 35 electrically conductive matrices, ${ }^{5}$ templates for cell growth or the growth of sol-gel structures, ${ }^{6}$ catalysis ${ }^{7}$ and in many other industrial fields such as cosmetics, oils, and foods. ${ }^{1}$ These hierarchical, self-assembled, and viscoelastic materials may be considered to be either hard or soft based on their rheological 40 characteristics. Among different classification criteria, that according to the driving forces for molecular aggregation is probably the most familiar. Thus, the two major groups are constituted by chemical gels ${ }^{8,9}$-based on covalent bonds, usually cross-linked polymers-, or physical gels ${ }^{1,10,11}$-made of either low45 molecular-weight (LMW) compounds or polymers, and based on non-covalent bonds, predominantly hydrogen-bonding, van der Waals, charge-transfer, dipole-dipole, $\pi-\pi$ stacking, and coordination interactions, which usually lead to reversible gel-tosol phase transitions-. Furthermore, systems based on both types 50 of connections are also known. ${ }^{12,13}$ The solid-like appearance of gel materials is the result of the entrapment of the liquid (major component) into the compartments of a solid 3D-matrix of a large surface area (minor component), typically through surface tension 
and capillary forces. ${ }^{14,15}$ The formation of the 3D-network through a number of "junction zones" 16 is a consequence of the entanglement of 1D-polymeric (or suprapolymeric) strands of macromolecules (or LMW molecules), -so called gelators-, ${ }^{1}$ 5 typically of micrometer scale lengths and nanometer scale diameters. ${ }^{17}$ Hence, in many cases, gels may immobilize up to $10^{5}$ liquid molecules per gelator and increase the viscosity of the medium by a factor of $10^{10}$, with the potential to respond to a variety of external stimuli. ${ }^{18,19}$

10 Despite the extensive and remarkable achievements of supramolecular chemistry in many controlled self-assembly processes, ${ }^{20}$ most of LMW gelators have been found by serendipity rather than rational design. Thus, the control of gelation phenomena and the design of new gelators are still ${ }_{15}$ challenging tasks, ${ }^{21}$ in particular when multiple dynamic noncovalent interactions (e.g. gelator-gelator, gelator-solvent, solvent-solvent, gelator aggregates-solvent) ${ }^{22}$ are taken into account. In this regard, gelators based on organic ammonium are interesting mainly due to their flexible and versatile hydrogen

20 bond pattern, which allows control of the dimensionality of the network. ${ }^{23}$ On the other hand, organic ammonium salts have been recognized as structurally simple supramolecular synthons, ${ }^{24}$ which could permit the fine-tuning of the non-covalent molecular aggregation mode by the incorporation of selected functional 25 groups. In comparison with the conventional one-component gelators, the two-component ammonium-based systems provide additional interest because multicomponent systems allow a further complexity of the hierarchical self-assembling process to form higher aggregates, which are responsible for the microphase 30 separation in the gel state. ${ }^{17,25}$

In this article, we report on the discovery and study of a new synergistic multicomponent organogelator liquid system (MGS), which shows intriguing gelation properties and exemplify the tough competition existing between gelation and crystallisation 35 phenomena. The MGS is formed by a methanolic solution of $(1 R, 2 R)$-1,2-diaminocyclohexane L-tartrate and hydrochloric acid (Figure 1).

40

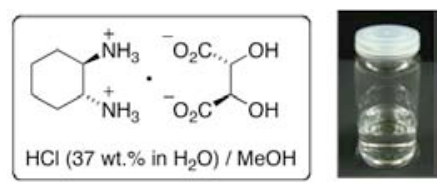

45 Fig. 1 Left: Components of the multicomponent organogelator liquid system (MGS). Right: Digital photograph of a glass vial containing the transparent solution prepared under the optimal molar ratio $(1 R, 2 R)-1,2$ diaminocyclohexane L-tartrate:MeOH:HCl: $\mathrm{H}_{2} \mathrm{O} \approx 1: 193: 2.4: 8.3$

50 Several features make this system unique in comparison with other organogelators, especially those based on ammonium carboxylates: ${ }^{23}(1)$ it is a multicomponent solution, in which each component and its stoichiometry plays a key role in the reproducible formation and stabilization of the organogels; (2) it

${ }_{55}$ is formed by simple, small, and commercially available chiral building blocks (dissolved in a well-defined solvent system: $\mathrm{MeOH} / \mathrm{HCl} / \mathrm{H}_{2} \mathrm{O}$ ), which are easily amenable for further modifications; (3) the gelation phenomenon takes place efficiently at low temperature upon warming up the isotropic ${ }_{60}$ solution, conversely to the typical gel preparation protocol (i.e. heating-cooling process); (4) the formed organogels are not thermoreversible despite the non-covalent interactions that characterize the 3D-network.

\section{Experimental $^{26}$}

\section{${ }_{65}$ Materials}

Unless otherwise noted, all organic compounds, mineral acids and anhydrous solvents were purchased from commercial suppliers and used without further purification. The manipulation of $\mathrm{HCl}$-containing solutions must be carried out accurately by the 70 use of precision glass syringes or pipettes, ensuring proper cleaning after each use.

\section{Preparation of optimal MGS}

Typically, a $0.3 \mathrm{M} \mathrm{HCl} / \mathrm{MeOH}$ stock solution was prepared using $\mathrm{HCl} 37$ wt.\% in aqueous solution and dry $\mathrm{MeOH} .(1 R, 2 R)-1,2-$ 75 Diaminocyclohexane L-tartrate $(254 \mathrm{mg}, 0.96 \mathrm{mmol})$ was weighted in a glass vial and dissolved in $7.5 \mathrm{~mL}$ of the above $\mathrm{HCl} / \mathrm{MeOH}$ stock solution (2.4 equivalents of $\mathrm{HCl}$ respect to the tartrate salt). The organogelator solution prepared under these conditions is ca. $0.13 \mathrm{M}$ (referred to tartrate salt), transparent, 80 colourless and does not contain any visible solid particles. This guarantees the stoichiometry of each component, which is imperative for the formation of reproducible gel materials.

\section{Preparation of MGS-based gels}

Typically, the appropriate solvent $(1 \mathrm{~mL})$ was placed into a glass ${ }_{85}$ screw-capped vial $(10 \mathrm{~cm}$ length $\times 1 \mathrm{~cm}$ diameter, $1 \mathrm{~mm}$ wall thickness) and cooled down close to the freezing temperature of the solvent. Depending on this temperature, the proper cooling bath composition (e.g. liquid nitrogen-acetone) was prepared in a Dewar flask and the above glass-vials immersed in the bath 90 during at least $5 \mathrm{~min}$ prior addition of the gelator system. The appropriate volume of MGS was subsequently added under gentle hand stirring of the vial, and the mixture kept at low temperature for $1 \mathrm{~min}$. After this time, the cooling bath was removed and the resulting clear homogeneous solution was let to warm up to room ${ }_{95}$ temperature allowing the formation of the gels. Unless otherwise indicated, the degree of gel formation was quantified after $30 \mathrm{~min}$ by turning the vial upside-down and collecting the non-gelled solvent.

\section{Characterization}

100 Nuclear magnetic resonance (NMR) spectra were recorded at 25 ${ }^{\circ} \mathrm{C}$ on Bruker Avance-300 instrument. Fourier transform infrared (FT-IR) spectra were recorded at room temperature using a Excalibur FTS 3000 FT-IR spectrometer (Biorad) equipped with a single reflection ATR (attenuated total reflection) accessory 105 (Golden Gate, Diamond). Differential scanning calorimetry (DSC) measurements were performed in a Perkin-Elmer DSC7. The DSC thermograms were obtained under dynamic nitrogen atmosphere (gas flow rate $=20 \mathrm{~mL} \mathrm{~min}^{-1}$ ) at a heating rate of 3 ${ }^{\circ} \mathrm{C} \mathrm{min}{ }^{-1}$. Samples were placed on open aluminium pans (Perkin110 Elmer). An empty sample holder was used as reference and the runs were performed by heating the samples from 25 up to 60 $160{ }^{\circ} \mathrm{C}$, depending on each sample. The values were reported as the average of two independent measurements. Field Emission Scanning Electron Microscopy (FESEM) images of xerogels 
(freeze-dried gel samples) were obtained with a Zeiss Merlin, Field Emission Scanning Electron Microscope operated at an accelerating voltage of $10 \mathrm{kV}$. The samples were sputtered (40 $\mathrm{mA}, 60$ seconds) with $\mathrm{Pt}$ (film thickness $\sim 10 \mathrm{~nm}$ ) before imaging by a SCD500 Leica EM. Atomic force microscopy (AFM) was performed using Tapping Mode (INTREGA Probe Nanolaboratory, NT-MDT) at $1 \mathrm{~Hz}$ scanning rate with a TiN (NT-MDT Nanoprobe tip NSG01 series) tip at drive frequency $115-190 \mathrm{kHz}$ (force constant 2.5-10 N/m). Dynamic rheological 10 measurements were carried out using an advanced rheometer AR 2000 (TA Instruments) equipped with a cooling system (Julabo C). A $20 \mathrm{~mm}$ plain plate (stainless steel) geometry was used for the measurements. Strain sweep measurements were initially performed to estimate the $\%$ at which reasonable torque values 15 (ca. $\times 10$ resolution limit of the transducer) could be obtained. Subsequently, frequency and time sweep measurements were performed in dynamic mode. To prevent solvent evaporation over the period of the experiment and the appearance of artefacts, a thin layer of low viscosity oil was added on the top of the sample.

20 A Büchi GKR-50 Kugelrohr apparatus was used to estimate the gel destruction temperatures $\left(T_{\mathrm{d}}\right)$ and compare them with the DSC thermograms. An optical microscope (Wild Makroskop M420 1.25x) equipped with a digital camera (Canon Power shot A640) was used at 15.6x magnification to photograph the crystals 25 formed within the gel over time. XRD analyses were carried out with an Agilent Technologies Inc. SuperNova diffractometer. A microfocus copper source was used together with an Atlas CCD camera. The cell parameters were determined in six different goniometer orientations by omega scans. Stock solutions were 30 accurately prepared in volumetric flasks. Turbidity measurements were carried out at room temperature using a Camlab CW8100 Portable Turbidimeter calibrated with T-CAL standards and provided with an infrared LED as light source $(\lambda=860 \mathrm{~nm})$ and a photodiode scattered light detector at an angle of $90^{\circ}$. The

35 samples were vigorously shaken for $1 \mathrm{~min}$ before measurement ensuring the absence of bubbles. The vials were rotated in the sample chamber about $45^{\circ}$ after each measurement for each sample until the reading matches within \pm 0.01 nephelometric turbidity units (NTU). A VWR ${ }^{\mathrm{TM}}$ ultrasonic cleaner (USC200TH) 40 was used during the solubility tests of the tartrate salt. The temperature of the ultrasonic water bath was $33 \pm 2{ }^{\circ} \mathrm{C}$ after 30 min sonication. Statistical validation of results was performed by simple one-way analysis of variance yielding overall significance $(p<0.05)$. The values in the text, tables and figures are expressed 45 as mean \pm standard deviation.

\section{Results and discussion}

During the standard protocol of tartaric acid-mediated racemic resolution of ( \pm -trans-1,2-diaminocyclohexane, ${ }^{26,27}$ we found that one of the intermediate compositions, which contained so $(1 R, 2 R)$-1,2-diaminocyclohexane L-tartrate, $\mathrm{MeOH}$ and aqueous $\mathrm{HCl}$, showed a remarkable ability to instantaneously form jellylike materials upon contact with a variety of other organic solvents at room temperature. A comprehensive study of this phenomenon (vide infra) led to a ca. $0.13 \mathrm{M}$ methanolic solution 55 of $(1 R, 2 R)$-1,2-diaminocyclohexane L-tartrate and 2.4 equiv of concentrated $\mathrm{HCl}$ (37 wt.\% in aqueous solution) as the optimal MGS, which corresponds to a molar ratio of $(1 R, 2 R)-1,2-$ diaminocyclohexane L-tartrate:MeOH:HCl: $\mathrm{H}_{2} \mathrm{O} \approx 1: 193: 2.4: 8.3$. As it will be discussed later, the presence and stoichiometry of ${ }_{60}$ each component was found to play a key role on the gelation process.

\section{Determination of the optimal molar composition}

In order to determine the optimal molar composition of the gelator solution, we first carried out an extensive two-variables ${ }_{65}$ screening defined by the tartrate salt concentration and number of equivalents of $\mathrm{HCl}$. This study provided a 2D-rectangular matrix of 132 elements. ${ }^{26}$ In order to minimize the experimental error derived from multiple volume measurements, a $0.3075 \mathrm{M}$ stock $\mathrm{MeOH}-\mathrm{HCl}$ solution was prepared and used during the solubility 70 screening of the salt. The results demonstrated that at least 2.1 equivalents of $\mathrm{HCl}$ (respect to the $(1 R, 2 R)-1,2-$ diaminocyclohexane L-tartrate) was necessary to completely dissolve the salt and provide a transparent gelator solution without any visible residue (Figure 2). The experimental turbidity 75 values of these solutions were bellow $0.35 \pm 0.05$ NTU. All systems prepared using less than 2.1 equivalents of $\mathrm{HCl}$ afforded mixtures with visible remnants of undissolved material. From these samples, only the one containing 2.0 equivalents of $\mathrm{HCl}$ could be turned into a clear solution upon $1 \mathrm{~min}$ sonication. Thus, 80 the turbidity value of systems containing for example only 1.7 equivalents of $\mathrm{HCl}$ exceeded $1040 \pm 50 \mathrm{NTU}$ even after $30 \mathrm{~min}$ sonication or gently heating with a heat-gun. These solubility properties were routinely observed within a salt concentration range of 0.06 to $1.0 \mathrm{M}^{26}$

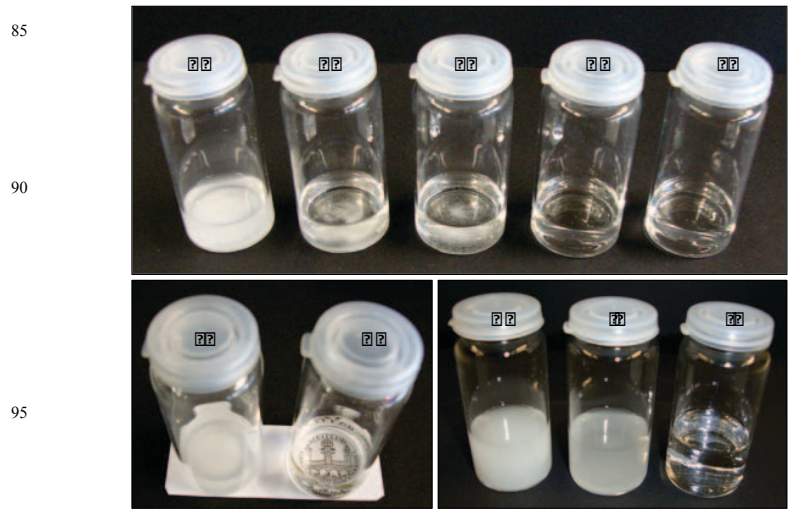

Fig. 2 Digital photographs showing the critical effect of the amount of $\mathrm{HCl}$ in the solubility of the tartrate salt in $\mathrm{MeOH}$ (ca. $0.13 \mathrm{M}$ ). The 100 mixtures in vials A-D were sonicated for $10 \mathrm{~min}$, and the pictures taken 30 min later: $\mathrm{A}=1.7$ equiv $\mathrm{HCl} ; \mathrm{B}=1.8$ equiv $\mathrm{HCl} ; \mathrm{C}=1.9$ equiv $\mathrm{HCl}$; $\mathrm{D}=2.0$ equiv $\mathrm{HCl} ; \mathrm{E}=2.4$ equiv $\mathrm{HCl}$ and without sonication. Vials $\mathrm{F}=$ A and $\mathrm{G}=\mathrm{E}$ were placed over the logo of the University of Regensburg, which is only visible through the latter solution $(2.4$ equiv $\mathrm{HCl})$. Vials $\mathrm{H}$ 105 (1.0 equiv $\mathrm{HCl}), \mathrm{I}(1.7$ equiv $\mathrm{HCl})$ and $\mathrm{J}(2.4$ equiv) were photographed after $30 \mathrm{~min}$ sonication and vigorous hand-shaken of the closed vials. Herein, the equiv of $\mathrm{HCl}$ are referred to the tartrate salt. Note that in the absence of $\mathrm{HCl}$ the tartrate salt remains insoluble in all tested solvents even after long periods of sonication and heat. ${ }^{26}$

110

The use of concentrations higher than $0.3 \mathrm{M}$ resulted in spontaneous slow growth of large monocrystals at room temperature in closed vials (Figure 3 ). NMR and single crystal XRD studies confirmed that these crystals correspond to $(1 R, 2 R)$ 115 1,2-diaminocyclohexane dihydrochloride. ${ }^{28}$ This finding has significant mechanistic implications that are discussed later in 
this paper.

A comparative study of the stability of various gels prepared using the clear solutions of the $2 \mathrm{D}$-matrix,${ }^{26}$ allowed to establish ca. $0.13 \mathrm{M}$ methanolic solution of tartrate salt containing 2.4 5 equivalents of $\mathrm{HCl}$ (respect to the salt) as the optimal composition of the MGS. No major changes in the gel properties were observed when they were prepared using MGS aged for about 7 days in a sealed vial and in dark. However, some detriment in the temporal stability of some gels could be observed 10 when MGS aged for longer periods of time were used.

It is important to remark that the obtention of reproducible gel materials depend, among other factors described later, on the use of stable and clear gelator solutions. This ensures not only a welldefined gelator concentration in each experiment, but also the

15 absence of any solid-particle nucleating agent that could favour the crystallisation of $(1 R, 2 R)$-1,2-diaminocyclohexane dihydrochloride, and consequently the destruction of the gel. Hence, the use of uncontaminated materials is also a major requirement during these experiments.

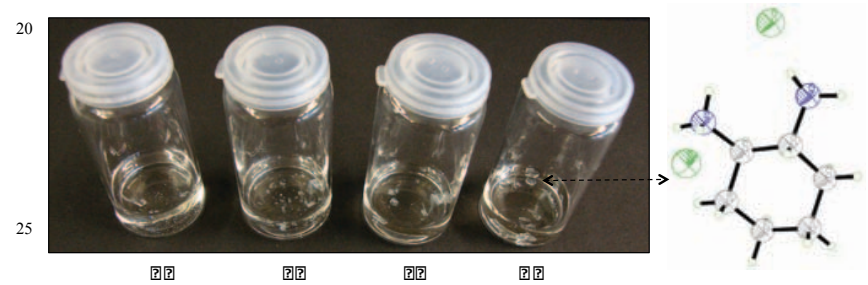

Fig. 3 Digital photographs showing the formation of monocrystals of $(1 R, 2 R)$-1,2-diaminocyclohexane dihydrochloride from MGS solutions prepared with 2.4 equiv $\mathrm{HCl}$ at different concentrations in $\mathrm{MeOH}$ : $\mathrm{A}=$ ${ }_{30} 0.25 \mathrm{M} ; \mathrm{B}=0.3 \mathrm{M} ; \mathrm{C}=0.5 \mathrm{M} ; \mathrm{D}=1.0 \mathrm{M}$. The appearance of the crystals was faster and more abundant at the higher concentrations. The ORTEP diagram on the right illustrates the crystal structure.

\section{Gel preparation}

The formation of gel-like materials upon addition of small 35 volumes of the MGS to a variety of solvents took place extremely fast at room temperature. ${ }^{23}$ However, such fast kinetics avoided the appropriate homogenization of the mixtures (MGS + solvent) before the gelation phenomenon, which caused no gelation of a significant amount of solvent. Under these conditions, the

40 formation of fluid-filled cavities within the non-homogeneous gel phase could be observed and disrupted upon mechanical stress (e.g. shaking or manipulation of gel with a spatula). Among many experimental trials, homogeneous organogels were formed when the solvents were cooled down close to theirs freezing points ${ }^{26}$

45 before addition of the MGS. By using this procedure the gelation kinetics could be dramatically slowed down, allowing the preparation of an isotropic solution containing MGS and the appropriate solvent, which further turned into homogeneous gel upon warmed up to room temperature (Figure 4). In general, we

${ }_{50}$ observed that a small portion of liquid remained non-gelled regardless the concentration of species (Figure 4, picture E). Thus, the degree of gel formation was quantified after gel formation by turning the vial upside-down and collecting the nongelled liquid (Table 1).
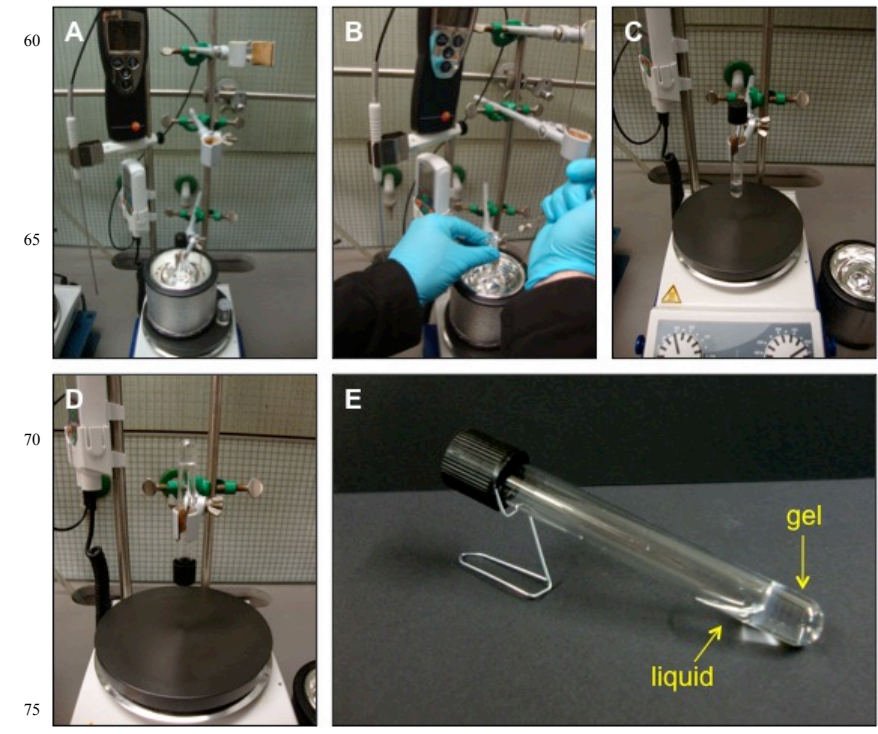

Fig. 4 Gel preparation steps. A: cooling down the test tube containing the desired solvent close to its freezing temperature; B: careful hand-shaking of the test tube and addition of the required amount of MGS at low temperature; C: removal of the cooling bath; D: gel state confirmed by ${ }_{80}$ "stable-to-inversion of the test tube" method; E: photograph of the transparent gel made in 1,4-dioxane showing the amount of non-gelled solvent (the picture was taken $30 \mathrm{~min}$ after removing the cooling bath).

Moreover, during this experimental procedure, we identified a 85 series of factors that have a significant influence on the gel formation. In this regard, the control of the solvent temperature (close to its freezing point), as well as a good stirring of the mixture [MGS + solvent] at low temperature could be used to regulate nucleation processes ${ }^{29}$ and afford the formation of most 90 stable and homogeneous gel materials. It is worth to mention that the glass vial design features (e.g. wall thickness, diameter) has also an effect on the gelation concentration, gelation kinetics and gel stability, since they can affect the stirring efficiency as well as the promotion of heat transfer through the vial, which would 95 directly influence the amount of energy transferred to the solvent mixtures.

\section{Solubility and gelation properties}

The gelation ability of the optimal MGS was evaluated for 40 solvents ${ }^{26}$ (Table 1). The state of the materials was examined by 100 the "stable-to-inversion of a test tube" method. ${ }^{26}$ As expected, the FT-IR spectra of both MGS and gel-based materials displayed the stretching vibration bands of both carboxylic (1710-1749 $\left.\mathrm{cm}^{-1}\right)$ and ammoniun (3250-2350 $\mathrm{cm}^{-1}$ ) groups. Hydrogen bond association bands in the range $3600-3200 \mathrm{~cm}^{-1}$ were also found 105 for the gel material. However, for accurate interpretations of the spectra it should be considered that alkane, water and methanol $\mathrm{O}-\mathrm{H}$ stretching bands are also overlapped in the above regions. ${ }^{26}$ Thus, no further conclusive information could be obtained from these spectra. The introduction of a $2 \mathrm{~mm}$ diameter thermocouple 110 into the solution (after removing the cooling bath) showed that in most cases the gelation process started well below $0{ }^{\circ} \mathrm{C}$. The gel condition of model gels was further confirmed by dynamic rheological measurements (vide infra). As shown in Table 1, the MGS was able to gel a variety of oxygenated and nitrogenated 
organic solvents at very low concentrations (entries 1-12). In contrast, no gel formation was observed with, among others, halogenated, aromatic and hydrocarbon solvents (entries 1315). ${ }^{26}$ The volume of MGS that afforded reproducible gel samples 5 (gelation volume, gv) was established in the range of 80-250 $\mu \mathrm{L}$, which corresponds to a gelation concentration range of ca. 0.30.9 wt. $\%$ referred to the tartrate salt. ${ }^{26}$

Table 1 Gelation ability of the optimal MGS in different solvents. ${ }^{a}$

\begin{tabular}{cccccccc}
\hline entry & $\begin{array}{c}\text { tested } \\
\text { solvent }\end{array}$ & $\begin{array}{c}\mathrm{gv} \\
(\mu \mathrm{L})^{b}\end{array}$ & $\begin{array}{c}T_{\text {mix }} \\
\left({ }^{\circ} \mathrm{C}\right)^{c}\end{array}$ & $\begin{array}{c}\text { non-gelled } \\
\text { liquid }(\mu \mathrm{L})^{d}\end{array}$ & $\begin{array}{c}T_{\mathrm{d}} \\
\left({ }^{\circ} \mathrm{C}\right)^{e}\end{array}$ & $\begin{array}{c}\text { aspect } \\
\text { phase }^{f}\end{array}$ & $\begin{array}{c}\text { temporal } \\
\text { stability }\end{array}$ \\
\hline 1 & NM & 100 & -20 & $40 \pm 20$ & $95^{h}$ & TG & $2-4 \mathrm{~h}$ \\
2 & ACN & 100 & -40 & $40 \pm 20$ & 62 & OG & $1-3 \mathrm{~d}$ \\
3 & BN & 150 & -10 & $240 \pm 50$ & 152 & TG & $1-2 \mathrm{~h}$ \\
4 & ACT & 250 & -90 & $110 \pm 20$ & 43 & OG & $2-5 \mathrm{~h}$ \\
5 & CHN & 150 & -10 & $115 \pm 20$ & $84^{h}$ & TG & $2-3 \mathrm{~h}$ \\
6 & MBN & 100 & -80 & $70 \pm 10$ & 68 & OG & $3-4 \mathrm{~d}^{i}$ \\
7 & DME & 100 & -50 & $40 \pm 20$ & 65 & OG & $1-4 \mathrm{~h}$ \\
8 & MEE & 100 & -60 & $60 \pm 10$ & 128 & OG & $3-4 \mathrm{~d}$ \\
9 & ETAC & 250 & -78 & $80 \pm 15$ & 44 & OG & $9-24 \mathrm{~h}$ \\
10 & THF & 150 & -78 & $40 \pm 20$ & 54 & TG & $4-5 \mathrm{~d}$ \\
11 & DEE & 180 & -100 & $90 \pm 30$ & 52 & OG & $3-4 \mathrm{~d}$ \\
12 & DOX & 80 & 13 & $50 \pm 30$ & 89 & TG & $2-3 \mathrm{~d}$ \\
13 & Group I & - & - & - & - & M & - \\
14 & Group II & - & - & - & - & I & - \\
15 & Group III & - & - & - & - & $\mathrm{P}$ & -
\end{tabular}

${ }_{10}{ }^{a} 1 \mathrm{~mL}$ of solvent was used in each experiment. Solvents abbreviations: $\mathrm{ETAC}=$ ethyl acetate; THF $=$ tetrahydrofuran; $\mathrm{DME}=1,2-$ dimethoxyethane; $\mathrm{MEE}=2$-methoxyethyl ether; $\mathrm{DEE}=$ diethyl ether; $\mathrm{CHN}=$ cyclohexanone; $\mathrm{MBN}=3$-methylbutan-2-one; $\mathrm{ACN}=$ acetonitrile; $\mathrm{BN}=$ benzonitrile; $\mathrm{DOX}=1$,4-dioxane; $\mathrm{ACT}=$ acetone; $\mathrm{NM}$ $15=$ nitromethane. Group $\mathrm{I}$ solvents $=$ methylene chloride, 1,2 dichloroethane, carbon tetrachloride, dimethylformamide; dimethylacetamide, dimethyl sulfoxide, ethanol, $n$-hexane, benzene, toluene, ethylene glycol, dibutyl ether, $N$-methyl-2-pyrrolidone, water. Group II solvents $=$ chloroform, 1,2-dichlorobenzene, nitrobenzene $n$ 20 octane, glycerol, cyclohexane, tetrachloroethylene, carbon disulfide; Group III solvents = 1-butyl-3-methylimidazolium hexafluorophosphate (ionic liquid, [BMIM][PF6]), pyridine, methyl tert-butyl ether. ${ }^{b}$ Volume of MGS used. The minimun volume necessary to achieve gelation is around this value (see ESI). ${ }^{c}$ Temperature of mixing (cooling bath 25 temperature during the addition of MGS). ${ }^{d}$ Amount of non-gelled liquid collected $30 \mathrm{~min}$ after gel formation. The values represent the average of at least 6 randomized experiments. ${ }^{e}$ Gel destruction temperature observed by DSC (error $= \pm 2{ }^{\circ} \mathrm{C}$ ). ${ }^{f}$ Abbreviations: TG $=$ transparent or slightly translucent gel; $\mathrm{OG}=$ opaque gel; $\mathrm{M}=$ miscible; $\mathrm{I}=$ immiscible; $\mathrm{P}=$ 30 precipitate. In the case of methyl tert-butyl ether a turbid gel-like material was initially formed but broken into small pieces of gel-like material after inversion of the test tube. ${ }^{g}$ Stability of the gels over time when stored undisturbed in a vertical position at $23 \pm 2{ }^{\circ} \mathrm{C}$. The lack of stability was defined when the gel was fragmented or partially liquefied without 35 resistance to inversion of the test tube. Abbreviations: $d=$ days; $h=$ hours. ${ }^{h}$ In this case the $T_{\mathrm{d}}$ was determined by a Kugelrohr-based method (see ESI) with an apparent error of $\pm 5^{\circ} \mathrm{C}$ for $\mathrm{CHN}$ and $\pm 10{ }^{\circ} \mathrm{C}$ for NM. ${ }^{i}$ The colour of this gel changes from white opaque to dark yellow-green opaque after $1-2 \mathrm{~h}$. Stability was judged by the destruction of the 40 coloured gel (see ESI).

It has recently been established that Kamlet-Taft solvent parameters $^{30}$ accounts for specific interactions between solvent and gelator molecules. ${ }^{31}$ The $\alpha$ parameter defines the hydrogen 45 bond donor ability of the solvent, which is of special importance in $3 \mathrm{D}$ networks built by hydrogen bonding. The $\beta$ parameter represents the hydrogen bond acceptor ability and can be associated to the thermal stability of the gel, whereas the $\pi^{*}$ parameter accounts for the polarisability and plays a key role on 50 solvation processes that occur during the gelation phenomenon. However, in contrast to most of organogels reported in the literature so far, the systems described here represent one of the most remarkable examples of sol-to-gel-to-crystal phase evolution. ${ }^{32}$ In this sense, the unique location of these transient ${ }_{55}$ gels right on the border between dissolution and crystallisation could contribute to explaining the lack of significant correlation between solvent parameters (i.e. relative permittivity and KamletTaft parameters) and gel properties (i.e. thermal and temporal stability). ${ }^{26}$ Nevertheless, it was still possible to clearly ${ }_{60}$ differentiate the ability of the solvent to be gelled by the MGS in function of the Kamlet-Taft parameters fitted by a Gaussian function (Figure 5). Typically, the solvents with higher tendency to form relatively stable gels were those presenting, simultaneously, no hydrogen bond donor ability (e.g. $\alpha \sim 0.0$ ), ${ }_{65}$ intermediate hydrogen bond acceptor ability (e.g. $\beta \sim 0.4$ ), and moderate polarisability (e.g. $\pi^{*} \sim 0.6$ ). In contrast, solvents characterized by either lower or higher $\beta$ and $\pi^{*}$ values afforded precipitation or complete solubilisation of the mixture, usually regardless the $\alpha$ value.

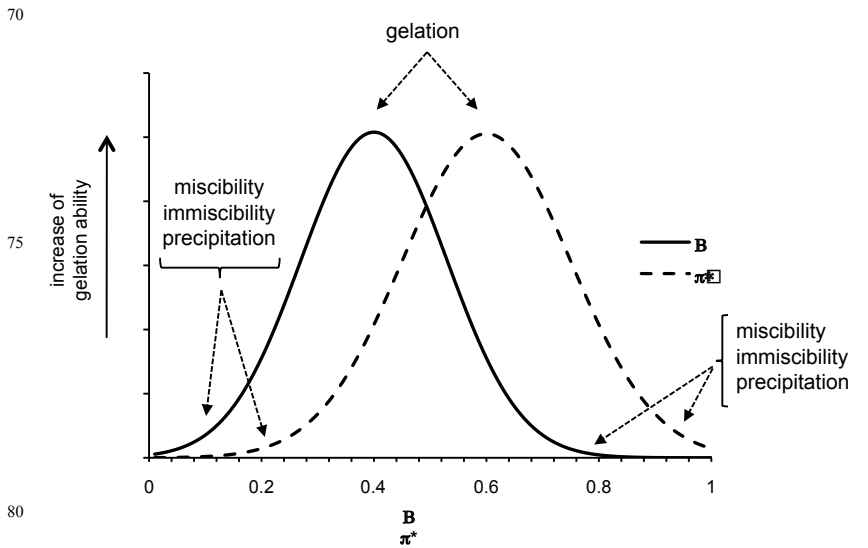

Fig. 5 Gaussian-like correlation between $\beta$ and $\pi^{*}$ solvent parameters and gelation ability of MGS. The model was built using 29 solvents.

Most of the formed organogels were off-white opaque with 85 different degrees of translucence (Figure 6), indicating the formation of aggregates smaller than $\lambda=400-700 \mathrm{~nm}$ (wavelength range of visible light). Only few gels (e.g. THF, DOX, BN, NM) were optically transparent or slightly turbid. ${ }^{26}$

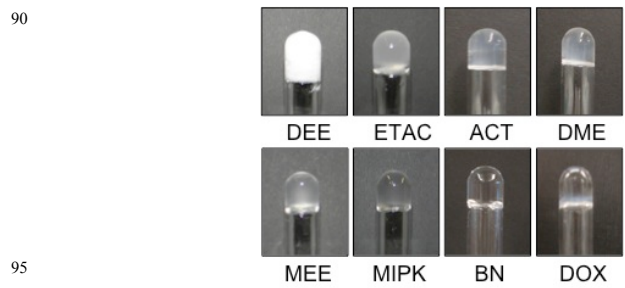

Fig. 6 Digital photographs of selected organogels made in various solvents showing different degrees of translucence.

During the evaluation of the temporal stability of the gels we 
were able to visualize a fascinating "arm wrestling" competition between gelation and crystallisation phenomena. The growth of $(1 R, 2 R)$-1,2-diaminocyclohexane dihydrochloride crystals (vide infra) inside the gel matrix was directly associated to the gel 5 weakening over time (Figure 7). Thus, the temporal stability of the gels, ranged from hours to several days (Table 1), represented an evident signature of the crystallisation kinetics. In many cases visible small crystals inside the gel matrix could be observed after the first day, even in those cases where the bulk material resisted 10 the inversion of the test tube. It is important to mention that the non-gelled liquid presented in the samples should be removed as soon as possible, since it contributes to weaken the gels. The weak nature of the transient gels and the inherent crystallisation process make also possible to observe continue leaking of solvent 15 over time in some of the samples, even when the initial nongelled liquid is removed. Dynamic gel-crystal transitions have been previously related to Ostwald rule of stages. ${ }^{33}$

Connecting to existing systems, the growth of superior inorganic, organic and protein crystals within supramolecular gels 20 as media has been successfully used over decades ${ }^{34}$ and has recently attracted also significant attention as a versatile new tool in pharmaceutical polymorph screening. ${ }^{35}$
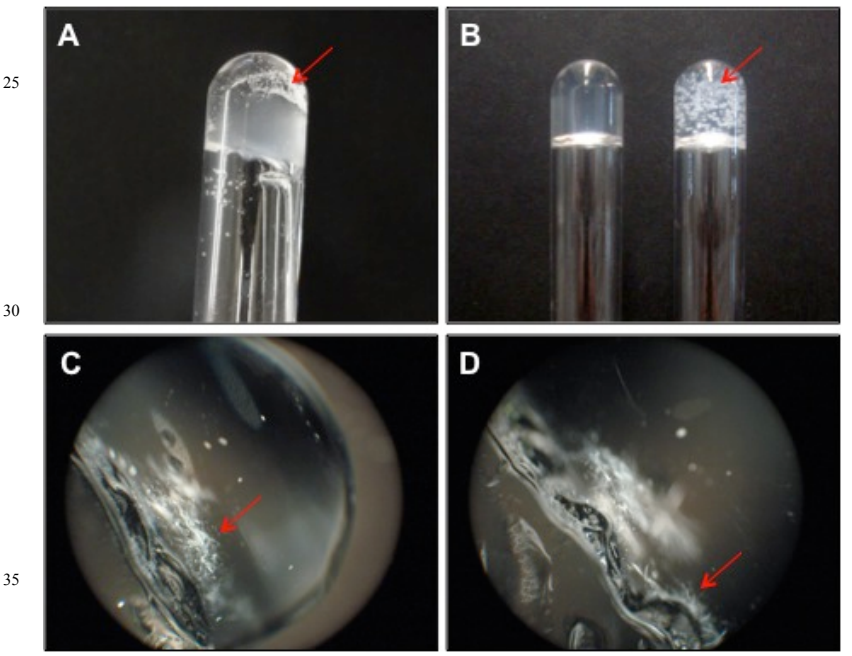

Fig. 7 Formation of crystals (arrows) inside the gel matrix. A: Partia destruction of the ACN-gel after crystallisation. B: Comparison between initial translucent ACN-gel and crystals-containing gel prepared with 200

$40 \mu \mathrm{L}$ of MGS. C-D: Optical microscope images of early crystals formed close to the interface gel-air (solvent = ETAC). Note: The bottom border of the test tube is observable in picture $\mathrm{C}$.

Very interestingly, the use of MGS prepared from $(1 R, 2 R)-1,2-$ 45 diaminocyclohexane and L-tartaric acid (ratio 1:1) instead of the preformed salt also afforded the formation of gels albeit with ca. 4-fold lower temporal stability.

\section{Thermal characterization}

Despite the non-covalent nature of these supramolecular gels, ${ }_{50}$ they did not present thermoreversibility. As it happens when the samples are submitted to a mechanical stress, heating the gels caused their destruction by inducing the formation of $(1 R, 2 R)$ 1,2-diaminocyclohexane dihydrochloride as a white precipitate, which is fully insoluble in the solvent medium and unable to get 55 back into the solution upon heating. This particular thermal behaviour made the "dropping ball method"36 inappropriate for the thermal characterization of these materials due to the lack of a clear transition to a liquid phase. Moreover, heating the samples caused in many cases a violent ejection of the solid material due 60 to the fast evaporation of the solvent trapped in the interstices. Although no reproducible values could be obtained using the "dropping ball method", DSC measurements (Figure 8) provided reliable information about the first endothermic transition, which was associated to the temperature at which the destruction of the ${ }_{65}$ gel network takes place $\left(T_{\mathrm{d}}\right){ }^{26}$

In contrast to most supramolecular gels, in which the gel-to-sol transition temperature clearly increases with the concentration of the gelator, the $T_{\mathrm{d}}$ in our materials was only slightly enhanced with the amount of MGS used in the experiments. ${ }^{26}$ However, ${ }_{70}$ DSC was sensitive enough not only to detect this enhancement but also the weakening of the material (decrease of the $T_{\mathrm{d}}$ ) when the amount of MGS induced the above-mentioned crystallisation process. For instance, the gel made in 1,4-dioxane showed the first endothermic transition at ca. 81,84 and $88^{\circ} \mathrm{C}$ using 40,50 75 and $75 \mu \mathrm{L}$ of MGS respectively. However, the use of higher amounts of MGS, i.e. $100 \mu \mathrm{L}$, destabilized the gel phase decreasing the $T_{\mathrm{d}}$ value until ca. $83{ }^{\circ} \mathrm{C} .{ }^{26}$

80

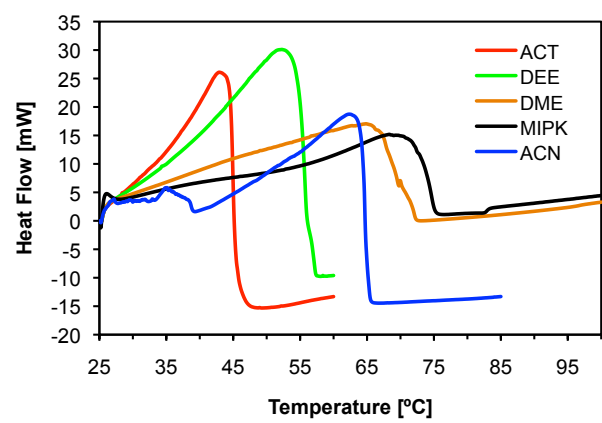

Fig. 8 DSC thermograms of different gels (for clarity, only a selection is 90 shown here. Additional spectra can be found in the ESI). Each DSC plot represents the average of two random measurements of gel samples prepared independently.

Influence of the components in the gelation ability of the MGS

${ }_{95}$ Considering that chirality doubtless plays a central role in the functional property of gel-based materials, ${ }^{37}$ and that our MGS is formed by chiral building blocks, we decided to investigate the effect that the enantiomeric purity of 1,2-diaminocyclohexane tartrate salt could have on the gel formation. All materials 100 obtained using non-enantiomerically pure MGS solutions (i.e. $0 \%$ $\leq$ salt ee $\leq 80 \%$ ) were unable to resist without flowing the inversion of the test tube, despite the formation of small pieces of jelly-like matter that clearly gained consistence upon increasing the optical purity of the tartrate salt. ${ }^{26}$ Stable-to-inversion of the 105 test tube gels were only obtained when enantiomerically pure MGS was used. As expected, enantiomeric MGS showed identical gelation properties. Moreover, MGS based on $(1 R, 2 R)$ 1,2-diaminocyclohexane D-tartrate yielded gels with much lower temporal stability than its diastereomeric counterpart when 110 prepared under identical experimental conditions, due to very fast crystals formation in the former. ${ }^{26}$

Moreover, we also studied the effect of the salt structure by replacing in the original MGS either the L-tartaric acid by 
different dicarboxylic acids derivatives or the $(1 R, 2 R)-1,2-$ diaminocyclohexane by alternative diamines. However, either turbid gelator solutions or non-stable gels were obtained with other diamines. ${ }^{26}$ Nevertheless, the gelator system showed a 5 higher tolerance towards the structure of the diacid counterpart. Thus, transient gels in different organic solvents could be obtained using MGS based on diethyl L-tartrate, succinic acid, adipic acid, dibenzoyl L-tartrate and L-(-)-malic acid. However, the temporal stability of these gels was at best half of that made 10 with the MGS based on $(1 R, 2 R)$-1,2-diaminocyclohexane and Ltartaric acid under the same conditions. ${ }^{26}$ Among the different acids, the gels with higher stability (ca. 1 day) were obtained with dibenzoyl L-tartrate and L-(-)-malic acid, which pointed out the importance of both carboxylic acids groups (somewhat higher

15 than the hydroxyl groups) and a chiral moiety for the formation of relatively self-standing gel networks.

In addition, a series of experiments demonstrated that each component presented in the optimal MGS was necessary for the formation of relatively stable gels. Thus, the use of a solution ${ }_{20}$ prepared in the absence of L-tartaric acid (i.e. $(1 R, 2 R)-1,2-$ diaminocyclohexane $+\mathrm{MeOH}$ (ca. $0.13 \mathrm{M}$ in diamine) $+\mathrm{HCl} 37$ wt. $\%$ in aqueous solution) resulted in the formation of either very weak partial gel-like materials or precipitates in less than $1 \mathrm{~h}^{26} \mathrm{In}$ the other hand, the replacement of $\mathrm{MeOH}$ by other alcohols like ${ }_{25} \mathrm{EtOH}, i$-PrOH or $n$ - BuOH did not allow to dissolve the tartrate salt not even in the presence of 2.4 equiv of $\mathrm{HCl}$.

Concerning the role of the mineral acid, the use of $48 \% \mathrm{HBr}$ instead of $37 \% \mathrm{HCl}$ also allowed the formation of gels albeit with much lower temporal stability (ca. 80\% lower than with the 30 optimal MGS) due to the faster formation of crystals. ${ }^{26}$ Remarkably, the amount of water in the MGS provided by the use of 37 wt.\% aqueous $\mathrm{HCl}$ was found to be necessary for the formation of gels with longer temporal stability. In the other hand, the presence of too much water (e.g. addition of 1 extra 35 equivalent of water) induced the crystallisation of $(1 R, 2 R)-1,2-$ diaminocyclohexane dichloride in only $1 \mathrm{~h}^{26}$

\section{Microscopy studies}

Scanning electron microscopy (SEM) imaging was used to gain insight into the microstructure of the aggregates in different 40 solvents. The existence of the metastable gel state very close to a crystalline phase was somehow also supported by the SEM images of high aspect ratios (Figure 9). A highly entangled fibrilar-like morphology could be distinguished in the freezedried gel derived from the gel in 1,4-dioxane (Figure 9D).

${ }_{45}$ However, most of the samples were characterized by the presence of aligned basalt-like (Figures 9C, F-H) or cobbled paved-like densely packed structures (Figures 9A-B, J), depending on the solvent used to prepare the gels. In general, the former structures presented uniform diameters of 2-3 $\mu \mathrm{m}$, whereas the latter ones so were differentiated with large cluster aggregates of $20-40 \mu \mathrm{m}$ in diameter.
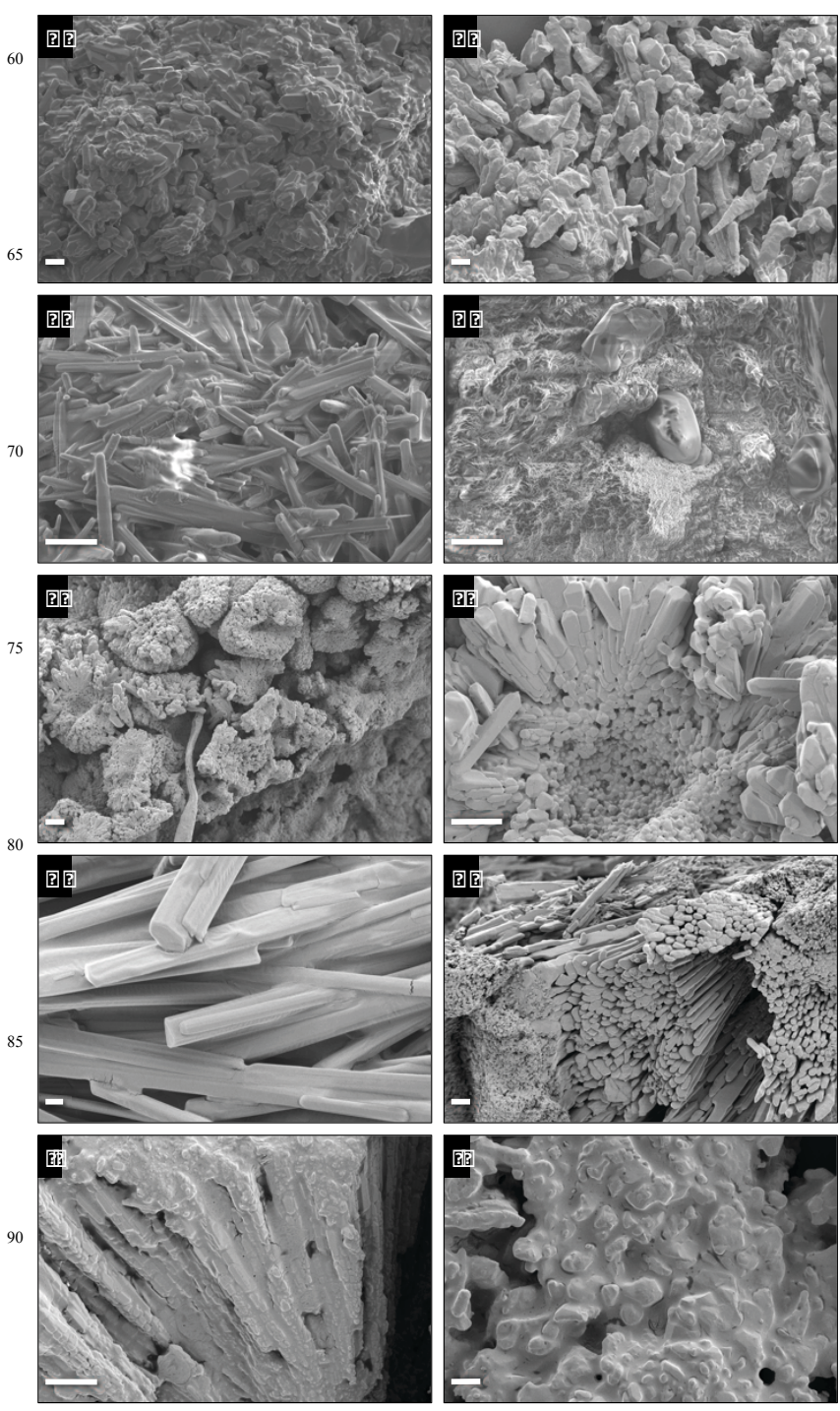

95 Fig. 9 SEM images of xerogels prepared from the gels made in different solvents as depicted in Table 1. A: gel made in ETAC, scale bar $=20 \mu \mathrm{m}$; B: gel made in DME, scale bar $=20 \mu \mathrm{m}$; $\mathrm{C}$ : gel made in THF, scale bar = $10 \mu \mathrm{m}$; D: gel made in DOX, scale bar $=10 \mu \mathrm{m}$; E: gel made in $\mathrm{BN}$, scale bar $=20 \mu \mathrm{m} ; \mathrm{F}$ : gel made in BN, scale bar $=10 \mu \mathrm{m} ; \mathrm{G}$ : gel made in ACN, 100 scale bar $=1 \mu \mathrm{m}$; $\mathrm{H}$ : gel made in $\mathrm{ACN}$, scale bar $=20 \mu \mathrm{m}$; I: gel made in MIPK, scale bar $=10 \mu \mathrm{m}$; J: gel made in DEE, scale bar $=30 \mu \mathrm{m}$.

To be certain that the observed structures were not artefacts, we also took atomic force microscopy (AFM) 3D images of the 105 surface topography, which were in agreement with the SEM images (Figure 10). In general, the photographs showed aligned mountain-range-shaped surfaces with average heights in the range of 25-100 nm and apparent diameters of about 100-200 $\mathrm{nm}$ for the largest individual feature. ${ }^{26}$ The lower limit was not 110 considered significant as it is dictated by the size of the AFM tip. 


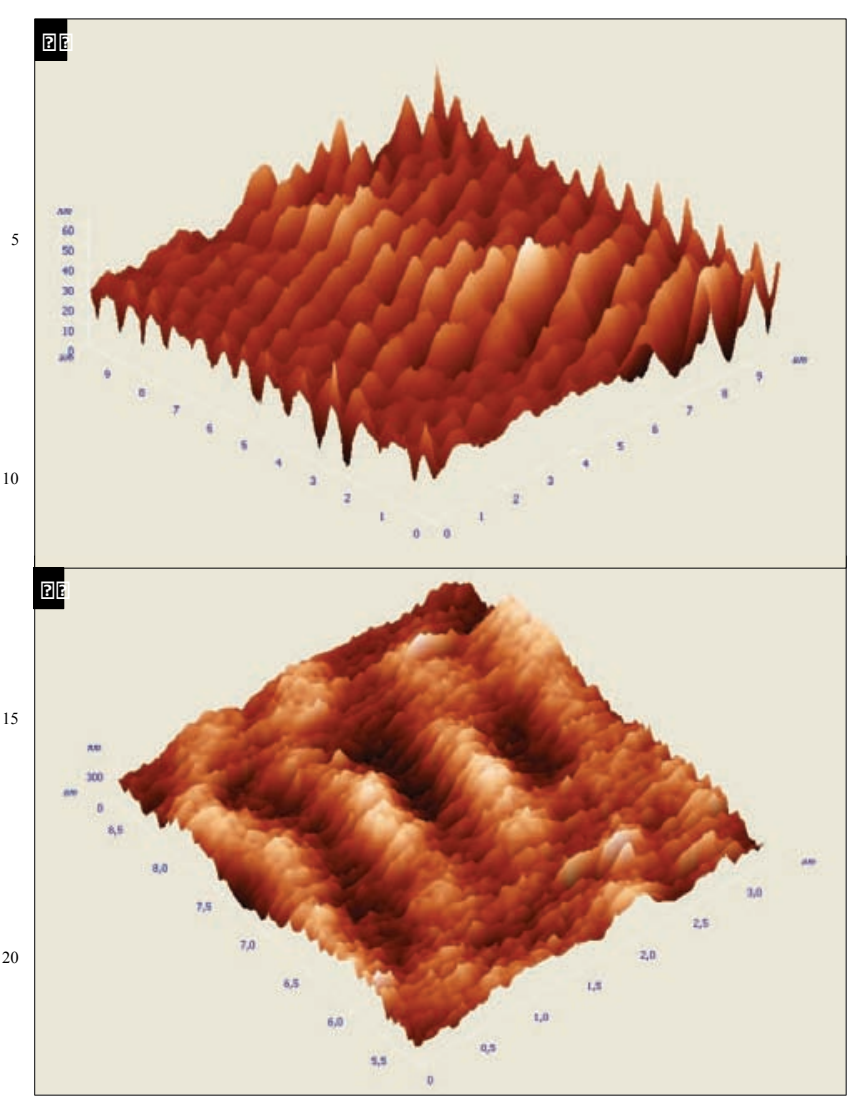

Fig. 10 3D-Surface topographic AFM images. A: $10 \times 10$ microns AFM 25 image of the xerogel prepared from the gel in BN; B: $8.5 \times 3$ microns AFM image of the xerogel prepared from the gel in ETAC.

\section{Dynamic rheological measurements of model gels}

We used oscillatory rheological measurements to confirm the actual gel state of the short-lived materials (storage modulus $G^{\prime}>$

${ }_{30}$ loss modulus $G^{\prime \prime}$ ) and evaluate their mechanical response within a linear viscoelastic regime of strain and frequency. Dynamic frequency sweep (DFS) and dynamic strain sweep (DSS) experiments highlighted the viscoelastic and fragile nature of the gels, which were destroyed at low frequency in the range of 1${ }_{35} 8 \%$ strain (Figure 11, top). A considerable angular frequency dependence of the solid modulus was more evident for the gels that showed also lower temporal stability. Further dynamic time sweep (DTS) experiments, at $0.1 \%$ strain and $\omega=1 \mathrm{~Hz}$ frequency) showed that $G^{\prime \prime}$ was about one order of magnitude 40 lower than $G^{\prime}$ indicating that the gels presented poor flexibility (Figure 11, bottom). Moreover, the plot as a function of ageing time showed a fast decrease of the both $G^{\prime}$ and $G^{\prime \prime}$ at almost the same rate. After only 10 min the solid modulus showed already a dramatic reduction (over $70 \%$ of the initial value!), pointing out

45 the low tolerance of the gel matrix to the external shear force, which was in agreement with the easy disruption and liquid leakage of the transient gels observed for example during their manipulation with a spatula. As indicated by the rates of decrease in modulus, the mechanical damping properties of the materials ${ }_{50}$ did not suffer major changes during the first $10 \mathrm{~min}$ of the experiment (constant $\tan \delta\left(G^{\prime \prime} / G^{\prime}\right)$ value $\sim 0.2-0.3$ ). However, a rise in the $\tan \delta$ value was evident as progressing the deterioration of the gel phase. ${ }^{26}$

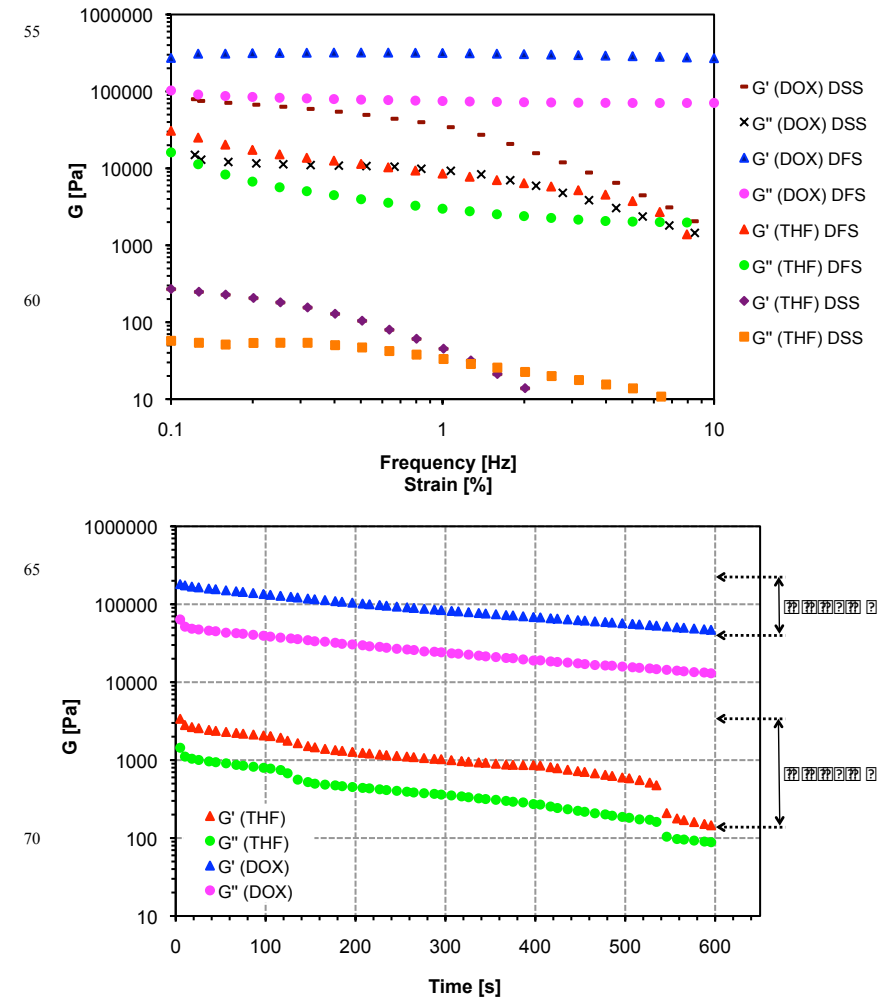

Fig. 11 DFS, DSS (top) and DTS (bottom) rheological measurements of 75 model transient gels prepared in THF and 1,4-dioxane.

\section{Mechanistic considerations}

The foregoing results have clearly demonstrated the formation of transient gels by using the described MGS based on $(1 R, 2 R)-1,2-$ diaminocyclohexane L-tartrate. Although the formed extended 80 non-covalent network is stable, it is not the thermodynamic minimum. A previous detailed crystallographic study of this salt demonstrated that it exhibits a highly interconnected bidirectional hydrogen-bonding polymeric network. The constitutional element of the supramolecular aggregate is a tweezer-shaped pattern 85 involving hydrogen-bonding interactions between ammonium nitrogen (donors) and hydroxyl oxygen (acceptors) (Figure 12). In this assembly the ammonium groups are hydrogen-bonded to carboxylate groups in one direction and to hydroxyl groups in the other. Such pattern of interactions needed for the development of 90 an extended network is determined by the defined chiral centers in the tartrate salt. Canal-like cavities delimited by the carboxylate, ammonium and hydroxyl groups are easily envisaged from the top view of the assembly. ${ }^{38}$

A plausible mechanism to explain the formation of the short${ }_{95}$ lived gel is indeed based on the compact nature of the tartrate salt 1. When $\mathbf{1}$ is suspended in $\mathrm{MeOH}$, the strongly hydrogen-bonded polymeric system does not interact efficiently with the solvent because of its inaccessibility. However, in the presence of aqueous $\mathrm{HCl}$, the ion-paired carboxylate-ammonium groups are 100 initially surrounded by chlorides ions (anion exchangers), water molecules and solvent molecules of the polar solvation shell. ${ }^{39}$ This situation accommodates a complex hydrogen-bonded network, involving all these species, which now becomes soluble in $\mathrm{MeOH}$ and stable enough to survive within a metastable phase, 
which slowly tends to evolve to the thermodynamic equilibrium position. Such energetic minimum is defined by the crystallization of the poorly soluble $(1 R, 2 R)-1,2-$ diaminocyclohexane dihydrochloride (4) -upon completion of the 5 ionic dissociation-exchange process- and the consequent release of soluble L-tartaric acid (3) (Figure 13). Nevertheless, when a small amount of the above methanolic solution (MGS 2) is mixed with another solvent that is a moderate hydrogen-bond acceptor, non-protic and less polar than $\mathrm{MeOH}$, the solubility of the 10 multicomponent aggregates (which acts as a supramolecular synthon $^{40}$ ) decreases but it is still hold within the dense hydrogenbonded network.

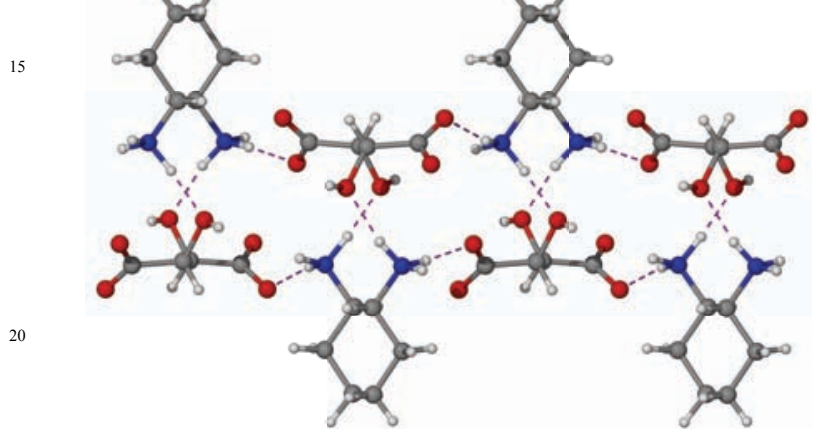

Fig. 12 Top view of the tweezer-shaped hydrogen-bonding between amino and hydroxy groups obtained from the crystallographic data of $25(1 R, 2 R)$-1,2-diaminocyclohexane L-tartrate.

In the above situation, an elusive balance of interactions between $\mathrm{MeOH}$ and $\mathrm{H}_{2} \mathrm{O}$ molecules and those of the organic nonprotic solvent should take place. This allows the interfacial 30 stabilization of the tartrate complex during enough time to permit its isotropic supramolecular assembly of limited solubility before the ionic exchange is completed (collapse of the gel and segregation of $\mathbf{4}$ as an amorphous precipitate -intergrown aggregates-). The exhaustive study carried out with several 35 multicomponent solutions designed within a $2 \mathrm{D}$ experimental matrix (salt concentration/equiv $\mathrm{HCl}$ ) has allowed us to recognize this intriguing equilibrium state at both microscopic and macroscopic scales.

As we have shown earlier, the crystallization rate of the 40 dichloride salt can be fine-tuned by, among other factors, the concentration of $\mathbf{1}$ and the amount of $\mathrm{HCl}$ presented in the mixture. Thus, a careful preparation of the MGS is necessary to ensure reproducible and optimal conditions for the formation of transient gels. Due to the strong hydrogen-bonding network of the 45 metastable gels, an additional energetic contribution (e.g. mechanical stress, heat) is required to accelerate the irreversible transition to the thermodynamically stable equilibrium position, which otherwise will simply take place over time. All the products were unequivocally characterized by NMR and ${ }_{50}$ elemental analysis, as well as by XRD in the case of crystalline 4 obtained from MGS $2{ }^{26}$

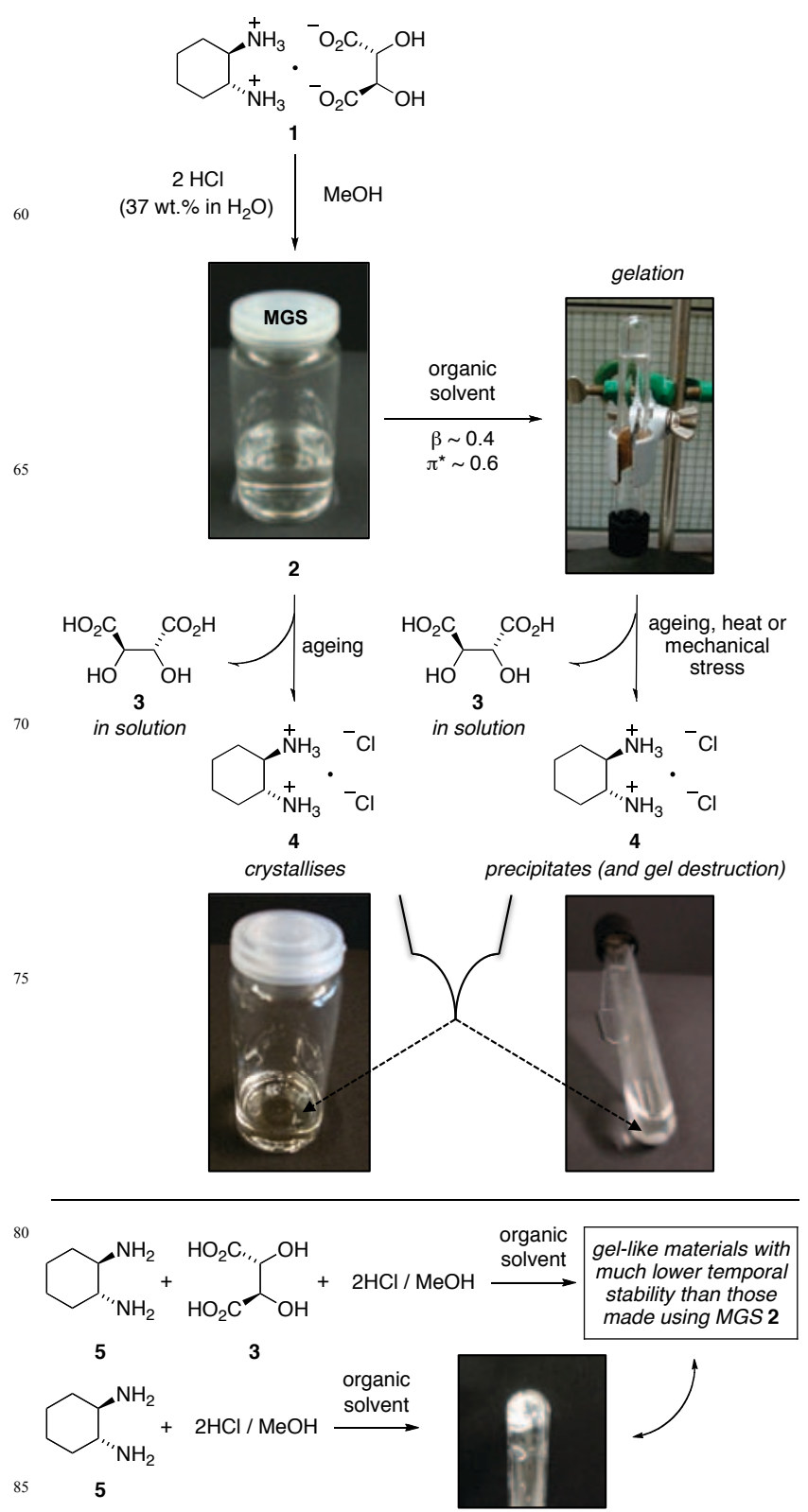

Fig. 13 Overview of MGS-induced crystallisation and gelation processes, and further destructive-evolution of the latter by precipitation of amorphous solids (top). Influence of (1) preformed ionic hydrogenbonded polymeric structure of the salt $\mathbf{1}$ and (2) the presence of tartaric 90 acid partner on the stability of the aggregates (bottom).

The exceptional ability of chiral trans-1,2-diaminocyclohexane to behave as an assembler of supramolecular hydrogen-bonding structures has been previously reported, ${ }^{41}$ and it is in perfect 95 agreement with our observations. Indeed, the proposed plausible gelation mechanism based on the $\mathrm{HCl}$-induced ionic dissociationexchange of $\mathbf{1}$ and the formation of a transient chloridecontaining assembly in solution, resembling that in the crystalline state, ${ }^{38,40}$ is supported by the series of experimental evidences 100 discussed along the different sections of this manuscript, namely:

(1) the formation of jelly-aggregates with much lower stability when either (a) the MGS was prepared using $(1 R, 2 R)-1,2-$ diaminocyclohexane and L-tartaric acid instead of its preorganized hydrogen-bonded polymeric salt $\mathbf{1}$, favouring a much 
faster formation of the dichloride salt 4; (b) the tartaric acid counterpart was eliminated from the multicomponent solution; (c) the use of aged MGS (which is most likely on its way to the equilibrium position); (d) the tartaric acid or the 5 diaminocyclohexane were exchanged by alternative diacids or diamines respectively (which can not replicate the strongly hydrogen-bonded, ion-paired situation of 1), or (e) nonenantiomerically pure MGS solutions were used.

(2) the thermodynamic formation of dichloride salt $\mathbf{4}$ as a 10 crystalline solid from the initial MGS or an amorphous solid after destruction of the gel phase;

(3) the delicate balance between the concentration of each component to maximize the half-life of the transient gels;

(4) the evidences of transient gelation even when highly 15 concentrated MGS solutions were used (despite the solubility limitations of the system)

\section{Conclusions}

The formation of supramolecular organogels is a result of a wellbalanced combination of numerous non-covalent interactions, 20 including those between gelator-gelator, gelator-solvent, aggregate-solvent and solvent-solvent molecules. Usually, a lack of control over these interactions caused an unpredictable competition between crystallization and gelation phenomena. The peculiar synergistic multicomponent gelator solution described in 25 this manuscript [ca. $0.13 \mathrm{M}(1 R, 2 R)$-1,2-diaminocyclohexane Ltartrate in $\mathrm{MeOH}+2.4$ equiv $\mathrm{HCl}$ (37 wt.\% in aqueous solution)] has showed a remarkable ability to kinetically frustrate a crystallization process allowing the evolution of transient gel aggregates at low temperatures and in a number of oxygenated 30 and nitrogenated solvents with moderate polarity. The foregoing results support a gelation mechanism based on the self-assembly of the tartrate salt resembling somehow that in the crystalline state. The experimental evidences point out a key role of the $(1 R, 2 R)$-1,2-diaminocyclohexane as the major structural director 35 of the assembly process, whereas the complementary tartaric acid partner acts as an important extender and stabilizer of the supramolecular network. The hydrochloric acid is involved in the solubilisation of the salt through a thermodynamic ionic dissociation-exchange process, which ends up with the formation 40 and segregation of $(1 R, 2 R)$-1,2-diaminocyclohexane dihydrochloride salt. As a consequence, an irreversible destruction of the gel takes place, which indicates the metastable nature of this phase that cannot be accessed from the thermodynamically equilibrated state. Despite the expected

45 disturbance of the hydrogen-bonding caused by the presence of methanol and $\mathrm{HCl}$, the real extension of such disruption is not yet clear in the MGS (which most likely denotes a highly organized hydrogen-bonded liquid system as observed in liquid water). In this sense, both MGS and its gels represent an extraordinary so example of persistence of memory of the parent assembly of $\mathbf{1}$ for a considerable length of time, showing similarities with the standard colloidal gels. ${ }^{42}$

It seems evident that the formation of the described transient gels depends on the ability to maintain a judicious balance 55 between the above-mentioned non-covalent interactions (specially anisotropic interactions between aggregate and solvent molecules) with different energetic contributions and a significant electrostatic component. ${ }^{43}$ In this sense, besides the chloridebridged transient assembly, the possibility of $\mathrm{MeOH}$-induced 60 microsolvation ${ }^{44}$ processes to allow the fine-tuning of the hydrogen-bonding pattern should be also considered. Moreover, it would be desirable to gain insights into a number of important physical-chemical aspects such as the exact mode of cation-anion association, the dissociation enthalpies of the critical bonds, the ${ }_{65}$ exact nature of the supramolecular nucleating species ${ }^{45}$ before crystallization of $\mathbf{4}$ or the interplay between the solvent system and the salt $\mathbf{1}$ in promoting the optimal ion-pairing for the stable self-assembly growth. Nevertheless, the significant complexity of this particular cooperative multicomponent liquid system makes 70 the performance of the required experimental/theoretical ${ }^{46}$ studies still a great scientific challenge.

Undoubtedly, the isolation and study of MGS-based gels provide an interesting perspective for the development of new gelators and a versatile platform for the study of pre-existing 75 interfaces during molecular crystallization. Moreover, we have recently found that some polar aprotic solvents like DMSO could, at least at some extent, replace $\mathrm{MeOH}$ in the gelator solution. Further investigations directed towards the detailed study of these new gelator solutions, extending the lifetime of the transient gels 80 based on calculated solubility parameters and Teas plots, controlling the kinetics of their structural transitions and understanding the dynamic of the hydrogen-bonded network are currently underway in our laboratories.

\section{Acknowledgements}

${ }_{85}$ I.K. ( $4^{\text {th }}$-year student at IISER, India) thanks DAAD (German Academic Exchange Service) for a 2011 WISE fellowship at the University of Regensburg (UR). J.B. thanks the research group E40 (Aminoacids and Peptides) for a pre-doctoral fellowship. The authors thank the Ministerio de Ciencia e Innovación 90 FEDER (project CTQ2010-17436) and Gobierno de Aragón (research group E40), CSIC (project PIE 200980I059) and UR (Anschubfinanzierung von Wissenschaftlichen Projekten-2011) for financial support. We are also indebted Dr. Manfred Zabel and Sabine Stempfhuber, Department of Crystallography (UR), ${ }_{95}$ for assistance with the XRD evaluations; Drs. R. Müller and A. Stoppa, Institute of Physical and Theoretical Chemistry (UR), for assistance with DSC measurements; and Prof. S. Hanessian (University of Montreal, Canada) for his input on this project.

\section{Notes and references}

1 D. J. Abdallah and R. G. Weiss, Adv. Mater., 2000, 12, 1237; P. Terech and R. G. Weiss, Chem. Rev., 1997, 97, 3133; J. H. van Esch and B. L. Feringa, Angew. Chem., Int. Ed., 2000, 39, 2263; O. Gronwald and S. Shinkai, Chem. Eur. J., 2001, 7, 4328; K. Hanabusa, Springer Ser. Mater. Sci., 2004, 78, 118; R. G. Weiss and P. Terech, Molecular Gels: Materials with Self-Assembled Fibrillar Networks; Springer: New York, 2006; M. George and R. G. Weiss, Acc. Chem. Res., 2006, 39, 489, and references therein.

2 A. Vintiloui and J.-C. Leroux, J. Controlled Rel., 2008, 125, 179; J. H. Jung and S. Shinkai, Top. Curr. Chem., 2004, 248, 223; N. M. Sangeetha and U. Maitra, Chem. Soc. Rev., 2005, 34, 821.

3 S. Li, V. T. John, G. C. Irvin, S. H. Bachakonda, G. L. McPherson and C. J. O’Connor, J. Appl. Phys., 1999, 85, 5965. 
4 T. Kato, Science, 2002, 295, 2414, and references therein.

5 J. Puigmarti-Luis, V. Laukhin, A. P. del Pino, J. Vidal-Gancedo, C. Rovira, E. Laukhina and D. B. Amabilino, Angew. Chem., Int. Ed., 2007, 46, 238; W. Kubo, S. Kambe, S. Nakade, T. Kitamura, K. Hanabusa, Y. Wada and S. Yanagida, J. Phys. Chem. B, 2003, 107, 4374.

6 C. Sanchez and M. Llusar, Chem. Mater., 2008, 20, 782; K. J. C. van Bommel and S. Shinkai, In Molecular Gels: Materials with SelfAssembled Fibrillar Networks; Springer: New York 2006; pp 857 893.

7 D. D. Díaz, D. Kühbeck and R. J. Koopmans, Chem. Soc. Rev., 2011, 40, 427.

8 T. Tanaka, Sci. Am., 1981, 244, 110.

9 D. Derossi, Y. Kajiwara and Y. Osada, Polymer Gels: Fundamentals and Biomedical Applications, Plenum Press, New York, 1991.

10 L. A. Estroff and A. D. Hamilton, Chem. Rev., 2004, 104, 1201; N. M. Sangeetha and U. Maitra, Chem. Soc. Rev., 2005, 34, 821; A Ajayaghosh, V. K. Praveen and C. Vijayakumar, Chem. Soc. Rev., 2008, 37, 109.

11 P. Xie and R. Zhang, J. Mater. Chem., 2005, 15, 2529; M. George, R. Mathew and R. G. Weiss, Molecular Gels, 2006, 449; D. K. Smith, Chem. Commun., 2006, 34.

12 S. M. Aharoni, in Synthesis, Characterization, and Theory of Polymeric Networks and Gels; Aharoni, S. M., Ed.; Plenum: New York, 1992.

13 E. R. Zubarev, M. U. Pralle, E. D. Sone and S. I. Stupp, Adv. Mater., 2002, 14, 198.

14 X. Huang, P. Terech, S. R. Raghavan and R. G. Weiss, J. Am. Chem. Soc., 2005, 127, 4336 .

15 F. Ilmain, T. Tanaka and E. Kokufuta, Nature, 1991, 349, 400, and references therein.

16 J. H. Shin, M. L. Gardel, L. Mahadeva, P. Matsudaira and D. A. Weitz, Proc. Nat. Acad. Sci., 2004, 101, 9636; Terech, P.; Furman, I. and Weiss, R. G. J. Phys. Chem., 1995, 99, 9558.

17 H. Ihara, M.Takafuji and Sakurai, T. Encyclopedia of Nanoscience and Nanotechnology 2004, 9, 473; Low Molecular Mass Gelators ed. Fages, F. in Topics in Current Chemistry, vol. 256, Springer-Verlag, Berlin, Heidelberg, 2005.

18 F. Ilmain, T. Tanaka and E. Kokufuta, Nature, 1991, 349, 400, and references therein.

19 Y. Osada and A. R. Khokhlov, Polymer Gels and Networks; Marcel Dekker: New York, 2002.

20 M. J. Serpe and S. L. Craig, Langmuir, 2007, 23, 1626; F. Diederich, Angew. Chem., Int. Ed., 2007, 46, 68; P. A. Gale, Chem. Soc. Rev. 2007, 36, 141 .

21 M. De Loos, B. L. Feringa and J. H. van Esch, Eur. J. Org. Chem., 2005,3615 .

22 Y. Wu, S. Wu, G. Zou and Q. Zhang, Soft Matter, 2011, 7, 9177; M. Bielewsky, A. Lapinsky, R. Luboradzki and J. Tritt-Goc, Tetrahedron, 2011, 67, 7222; W. Edwards, C. A. Lagadec and D. K. Smith, Soft Matter, 2011, 7, 110, and references therein.

23 K. Sada, T. Tani and S. Shinkai, Synlett, 2006, 15, 2364; D. R. Trivedi and P. Dastidar, Chem. Mater., 2006, 18, 1470, and references cited therein; M. Ayabe, T. Kishida, N. Fujita, K. Sada and Shinkai, S. Org. Biomol. Chem., 2003, 1, 2744.

24 G. R. Desiraju, Angew. Chem., Int. Ed., 1995, 34, 2311.

25 A. R. Hirst and D. K. Smith, Chem. Eur. J., 2005, 11, 5496.

26 See ESI for further information.

27 F. Gaslbol, P. Steenbol and B. S. Sorensen, Acta Chem. Scand., 1972, 26, 3605; P. J. Walsh, D. K. Smith and C. Castello, J. Chem. Educ., $1998,75,1459$

28 A. S. Abu-Surrah, T. A. K. Al-Allaf, M. Klinga and M. Ahlgren, Polyhedron, 2003, 22, 1529.

29 R.-Y. Wang, X.-Y. Liu, J. Narayanan, J.-Y. Xiong and J.-L. Li, J. Phys. Chem. B, 2006, 110, 25797.

30 M. J. Kamlet, J. L. M. Abboud, M. H. Abraham and R. W. Taft, J. Org. Chem., 1983, 48, 2877; R. M. C. Goncalves, A. M. N. Simoes, L. M. P. C. Albuquerque, M. Roses, C. Rafols and Bosch, E. J.
Chem. Res. (M), 1993, 1380; M. El-Sayed, H. Müller, G. Rheinwald, H. Lang, and S. Spange, Monatsh. Chem., 2003, 134, 361; A. Natrajan and D. Wen, Green Chem., 2011, 13, 913.

31 W. Edwards, C. A. Lagadec and D. K. Smith, Soft Matter, 2011, 7, 110.

32 J. De Rudder, B. Bergé and H. Berghmans, Macromol. Chem. Phys., 2002, 203, 2083.

33 V. J. Anderson and H. N. W. Lekkerkerker, Nature, 2002, 416, 811; P. Zhu, X. Yan, Y. Su, Y. Yang and J. Li, Chem. Eur. J., 2010, 16, 3176.

34 G. R. Desiraju; D. Y. Curtin and I. C. Paul, J. Am. Chem. Soc., 1977, 99, 6148; H. K. Henisch and J. M. Garcia-Ruiz, J. Cryst. Growth, 1986, 75, 203; C. Daiguebonne, A. Deluzet, M. Camara, K. Boubekeur, N. Audebrand, Y. Gérault, C. Baux and O. Guillou, Cryst. Growth Des., 2003, 3, 1015; R. I. Petrova and J. A. Swift, J. Am. Chem. Soc., 2004, 126, 1168; R. I. Petrova, R. Patel and J. A. Swift, Cryst. Growth Des., 2006, 6, 2709; H. Li, Y. Fujiki, K. Sada and L. A. Estroff, CrystEngComm, 2011, 13, 1060; D. Braga, S. d'Agostino, E. D'Amen and F. Grepioni, Chem.Commun., 2011, 47, 5154

35 J. A. Foster, M.-O. M. Piepenbrock, G. O. Lloyd, N. Clarke, J. A. K. Howard and J. W. Steed, Nature Chem., 2010, 2, 1037.

36 A. Takahashi, M. Sakai and T. Kato, Polym. J., 1980, 12, 335.

37 Z. Dzolic, K. Wolsperger and Zinic, M. New J. Chem., 2006, 30, 1411; A. Brizard, R. Oda and I. Huc, Top. Curr. Chem., 2005, 256, 167 , and references therein.

38 S. Hanessian, M. Simard and S. Roelens, J. Am. Chem. Soc., 1995, 117, 7630.

39 J. Gregory and M. J. Semmens, J. Chem. Soc., Faraday Trans. 1, 1972, 68, 1045 .

40 P. Sahoo, N. N. Adarsh, G. E. Chacko, S. R. Raghavan, V. G. Puranik and P. Dastidar, Langmuir, 2009, 25, 8742.

41 P. Dapporto, P. Paoli and S. Roelens, J. Org. Chem., 2001, 66, 4930, and references therein.

42 E. Zaccarelli, J. Phys. Condens. Matter 2007, 19, 323101.

43 D. Chandler, Nature, 2005, 437, 640.

44 A. M. Rijs, N. Sändig, M. N. Blom, J. Oomens, J. S. Hannan, D. A. Leigh, F. Zerbetto and W. J. Buma, Angew. Chem., Int. Ed., 2010, 49, 3896.

45 P. Jonkheijm, P. van der Schoot, A. P. H. J. Schenning and E. W. Meijer, Science, 2006, 313, 80.

46 D. J. Adams, K. Morris, L. Chen, L. C. Serpell, J. Bacsa and G. M. Day, Soft Matter, 2010, 6, 4144. 